CASE REPORT

D.S. Titelbaum

N.B. Sodha

M. Moonis

\title{
Transient Hemiglossal Denervation during Acute Internal Capsule Infarct in the Setting of Dysarthria-Clumsy Hand Syndrome
}

SUMMARY: A case of MR imaging-documented transient unilateral tongue denervation presenting during acute internal capsule infarction is described. Understanding the corticolingual pathway innervation of the hypoglossal nucleus is essential for explaining these findings. Awareness of the findings in this case will facilitate appropriate diagnosis, provide neuroanatomic explanation, and prevent misdiagnosis.

ABBREVIATIONS: DCHS = dysarthria-clumsy hand syndrome; TMS = transcranial magnetic stimulation

W hile permanent hypoglossal denervation is well known to occur subsequent to lower motor neuron injury, ${ }^{1}$ hypoglossal palsy due to supranuclear infarction has rarely been reported..$^{2-7}$ We report a case of transient reversible hemiglossal denervation edema on MR imaging, contralateral to acute internal capsule infarction in the clinical setting of DCHS. A neuroanatomic basis is discussed.

\section{Case Report}

A 73-year-old man with chronic hypertension presented with acute dysarthria and right arm weakness. Physical examination also revealed right facial droop and right arm incoordination. The tongue and uvula were midline without fasciculations or atrophy. The clinical diagnosis was DCHS. MR imaging performed at $1.5 \mathrm{~T}$ revealed a focal area of restricted diffusion at the posterior limb of the left internal capsule, indicating acute infarction (Fig 1A). Additionally, the right hemitongue was markedly diffusely swollen and hyperintense on T2-weighted imaging with right-sided posterior prolapse into the oropharynx (Fig 1B). Fatty atrophy was not identified on T1weighted images. The brain stem was normal.

Repeat MR imaging with gadolinium performed 2 days later to assess possible tongue mass revealed persistence of restricted diffusion in the posterior limb of the left internal capsule but with resolved right hemiglossal swelling and T2 hyperintensity and resolved posterior prolapse (Fig 2). There was no abnormal tongue enhancement or brain stem abnormality.

After 1 year, the patient had persistent ataxic right hemiparesis with spasticity. The tongue was midline with spasticity.

\section{Discussion}

Hypoglossal nerve dysfunction, often with tongue atrophy, is well known to occur secondary to lower motor neuron injury to the nerve or ganglion, with causes including brain stem

Received August 9, 2009; accepted after revision August 14.

From the Department of Radiology (D.S.T.), Shields Health Care, Brockton, Massachusetts; and Department of Neurology (N.B.S., M.M.), University of Massachusetts Medical Center, Worcester, Massachusetts.

Please address correspondence to David S. Titelbaum, MD, c/o Lorraine Abbene, Department of Radiology, Shields Health Care, 265 Westgate Dr, Brockton, MA 02301; e-mail: dtitelbaum@shields.com

Indicates open access to non-subscribers at www.ajnr.org

DOI 10.3174/ajnr.A1874 infarction, nerve invasion or compression by tumor or internal carotid artery dissection, and following injury during carotid endarterectomy or radical neck dissection surgery. MR imaging of acute hypoglossal denervation has been shown to produce edemalike hyperintensity on T2-weighted images, which may rapidly progress to fatty atrophy. ${ }^{1}$ Intramuscular T2 hyperintensity is commonly seen in the setting of lower motor neuron injury, may be detected as early as 24 hours following injury, ${ }^{8}$ and is thought to represent muscular edema from acute denervation due to fluid expansion of the extracellular space. ${ }^{9}$ Denervation has been shown to depolarize the resting potential of muscle cell membranes within 2 hours following injury due to inhibition of sodium-potassium pump function, thereby allowing increased membrane permeability of sodium and water. ${ }^{10-12}$ Muscle fiber is dependent on motor neuron integrity for maintenance of normal function and structure, mediated by sustaining action of electric impulse stimulation. ${ }^{11}$ As long as atrophy has not occurred, denervation or partial denervation is reversible if neuromuscular connections are re-established, resulting in recovery of membrane potential. $^{11}$

Corticolingual tract fibers (also referred to as corticohypoglossal tract or corticonuclear tract) extend from the precentral gyri through the corona radiata, internal capsules, and cerebral peduncles to the pontomedullary junction where some fibers decussate. ${ }^{6}$ Isolated dysarthria due to lacunar or cortical infarction is rare and is thought to be due to corticolingual tract dysfunction. ${ }^{2,7}$ DCHS has been reported to occur in $1.6 \%$ of patients with stroke and in $6 \%$ of those with lacunar infarcts. ${ }^{2}$ Commonly, supranuclear hypoglossal palsy is associated with ipsilesional arm weakness and supranuclear facial palsy, dysarthria, and dysphagia, presumably due to the close anatomic relationship of the respective motor pathways. ${ }^{6,7}$ While the hypoglossal nerve is a lower motor neuron, crossed and uncrossed upper motor neuron corticolingual fibers extend from both hemispheres to the hypoglossal nuclei. ${ }^{5}$ Following unilateral infarction, TMS of the affected hemisphere fails to produce an ipsilateral or contralateral lingual response, whereas TMS of the unaffected hemisphere produces a bilateral lingual response, even in the setting of unilateral lingual paralysis. ${ }^{5}$ Also, unilateral infarction inconsistently results in lingual paralysis and is presumably dependent on the ability of the unaffected hemisphere to activate uncrossed corticolin- 

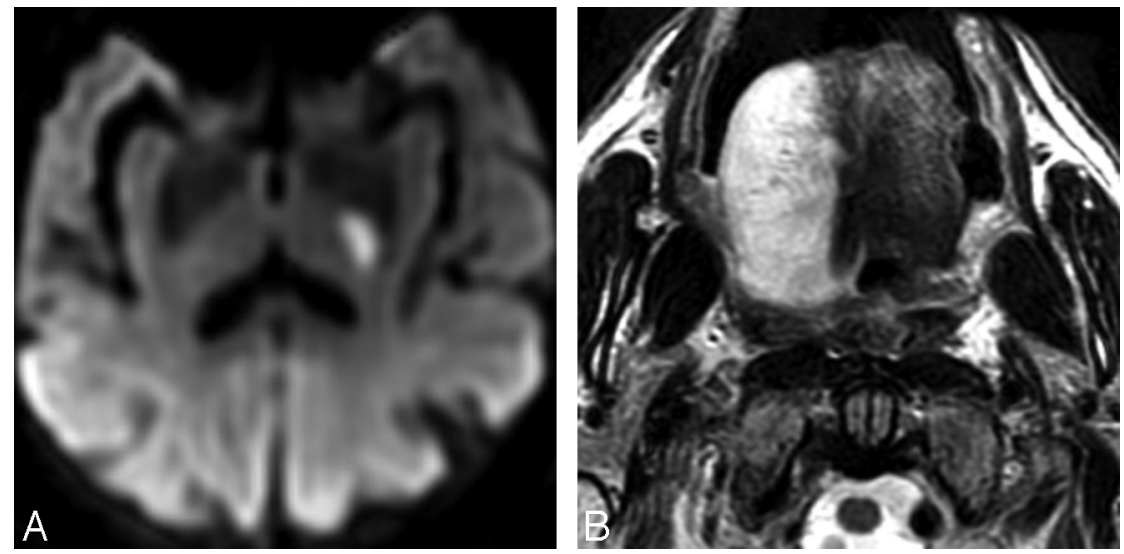

Fig 1. A 73-year-old man with acute onset of right arm weakness and dysarthria. A, Diffusion-weighted image $(b=1000)$ reveals focal hyperintensity at the posterior limb of the left internal capsule, indicating acute infarction. Hypointensity was present on an apparent diffusion coefficient map (not shown). B, Axial T2-weighted image obtained at the time of acute infarction shows diffuse right hemiglossal edema with swelling and posterior prolapse.

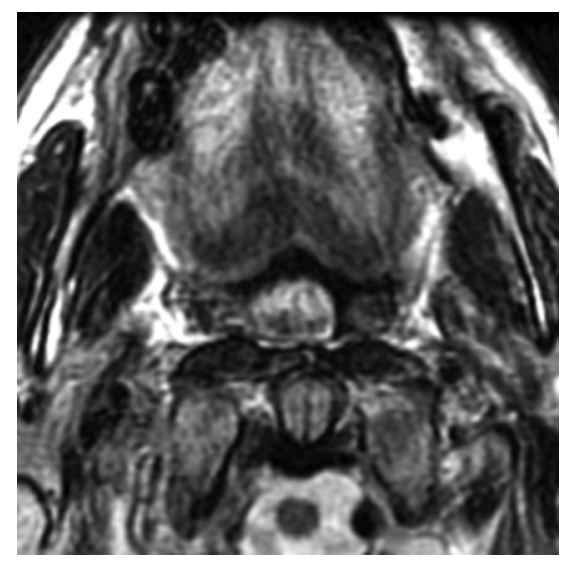

Fig 2. Axial T2-weighted image obtained 2 days later shows resolved right hemiglossal swelling and edema and resolved posterior prolapse.

gual projections. ${ }^{5}$ Corticolingual pathways are also variable between individuals, most commonly revealing bilateral cortical dependence, less commonly exclusive contralateral dependence, or rarely ipsilateral dependence. ${ }^{3}$

Tongue movement dysfunction is inconsistently seen in patients with dysarthria, even with abnormal corticolingual conduction measured by TMS, and is attributable to bilateral innervation. ${ }^{7}$ Even with motor impairment, physical findings may be masked by apraxia, because tongue deviation may only be detected at full protraction. ${ }^{3}$ Of additional note, despite the close anatomic proximity of the corticolingual and corticofacial motor tracts, corticofacial tracts are primarily contralateral, without bilateral uncrossed fibers, so that corticofacial infarction most often results in facial paresis. ${ }^{7}$

Unilateral findings in this case support the existence of predominant contralateral innervation from the motor cortex to the hypoglossal nucleus. Lack of tongue deviation on physical examination in this case could be explained by transient or incomplete denervation of the tongue that was in the process of recovering, preserved contralesional corticolingual innervation of the hypoglossal nucleus maintaining normal bilateral tongue function, or tongue apraxia preventing full tongue protraction, thus masking deviation.

Transient supranuclear tongue paralysis subsequent to unilateral internal capsule lacunar infarct is explainable by bilateral corticolingual innervation of the hypoglossal nucle- us. ${ }^{4,5}$ The transient nature of the tongue edema in this case presumably represented a reversible or compensated event that could be explained by reperfusion, by partial denervation with recruitment or re-establishment of latent neural pathways, or by transient voluntary inaccessibility of ipsilateral uncrossed corticolingual projections during the acute phase of the infarct. ${ }^{5,6}$

\section{Conclusions}

Hemiglossal denervation is not always due to lower motor neuron injury, can occur with supranuclear infarction, and can be transient and reversible. Awareness of the existence of individually variable bilateral crossed and uncrossed corticolingual projections facilitates a neuroanatomic explanation for these findings. In the future, one could postulate a potential role for diffusion tensor fiber tractography and functional MR imaging in the assessment of normal and impaired corticolingual pathways and lingual motor response.

\section{References}

1. Russo CP, Smoker RK, Weisman JL. MR appearance of trigeminal and hypoglossal motor denervation. AJNR Am J Neuroradiol 1997;18:1375-83

2. Arboix A, Bell Y, Garcia-Eroles L, et al. Clinical study of $\mathbf{3 5}$ patients with dysarthria-clumsy hand syndrome. J Neurol Neurosurg Psychiatry 2004;75:231-34

3. Durieu I, Leys D. Lingual motility in unilateral hemispheric vascular complications: study of the cortico-hypoglossal afferences [in French]. Rev Neurol (Paris) 1994;50:844-49

4. Kuypers HG. Corticobular connexions to the pons and lower brain-stem in man: an anatomical study. Brain 1958;81:364-88

5. Muellbacher W, Artner C, Mamoli B. Motor evoked potentials in unilateral lingual paralysis after monohemispheric ischaemia. J Neurol Neurosurg Psychiatry 1998;65:755-61

6. Umapathi T, Venketasubramanian N, Leck KJ, et al. Tongue deviation in acute ischaemic stroke: a study of supranuclear twelfth cranial nerve palsy in $\mathbf{3 0 0}$ patients. Cerebrovasc Dis 2000;10:462-65

7. Urban PP, Wicht S, Hopf HC, et al. Isolated dysarthria due to extracerebellar lacunar stroke: a central monoparesis of the tongue. J Neurol Neurosurg Psychiatry 1999;66:495-501

8. Bendszus M, Koltzenburg M, Wessig C, et al. Sequential MRI of denervated muscle: experimental study. AJNR Am J Neuroradiol 2002;23:1427-31

9. Polak JF, Jolesz FA, Adams DF. Magnetic resonance imaging of skeletal muscle: prolongation of $\mathrm{T} 1$ and $\mathrm{T} 2$ subsequent to denervation. Invest Radiol 1988;23:365-69

10. Albuquerque EX, Schuh FT, Kauffman FC. Early membrane depolarization of the fast mammalian muscle after denervation. Pflugers Arch 1971;328:36-50

11. MacIntosh BR, Gardiner PF, McComas AJ. Skeletal Muscle Form and Function. 2nd ed. Champaign, Illinois: Human Kinetics Publishers; 2006

12. Wareham AC. Effect of denervation and ouabain on the response of the resting membrane potential of rat skeletal muscle to potassium. Pflugers Arch 1978;373:225-28 